Año 13.

Revista de Investigación Académica sin Frontera Núm. 33 ISSN: 2007-8870 https://revistainvestigacionacademicasinfrontera.unison.mx/index.php/RDIASF/issue/view/22

Recibido el 23 de mayo de 2020. Dictaminado mediante arbitraje favorablemente 20 de diciembre de 2020.

\title{
Satisfacción de trabajadores en una organización pública educativa que presta servicios deportivos.
}

\section{Satisfaction of workers in a public educational organization that provides sports services.}

\begin{abstract}
Marina Reyes Robles ${ }^{1}$, Jesús Alfredo Rojo Villa ${ }^{2}$, Luis Ernesto López Esquerra ${ }^{3}$, Daniel Armando Sánchez Félix ${ }^{4}$

${ }^{1}$ Docente. Licenciatura en Entrenamiento Deportivo. Universidad Estatal de Sonora. México. (marina.reyes@ues.mx); http://orcid.org/0000-0002-0313-6056

2 Docente. Licenciatura en Entrenamiento Deportivo. Universidad Estatal de Sonora. México. (alfredo.rojo@live.com); https://orcid.org/0000-0003-3418-9158

3 Docente. Departamento de Deportes. Instituto Tecnológico de Sonora. México. (luis.lopez@itson.edu.mx); https://orcid.org/0000-0002-3312-8284

${ }^{4}$ Estudiante. Licenciatura en Entrenamiento Deportivo. Universidad Estatal de Sonora. México. (danielarmandosanchezfelix@gmail.com); https://orcid.org/0000-0002-8589-1126
\end{abstract}

\section{Resumen}

La satisfacción en la actualidad ha tomado especial importancia dentro de las organizaciones, todo lo relacionado al trabajador, por ello es considerado como un indicador de calidad dentro de la gestión, estando relacionado con la eficiencia de los servicios que ofrecen. El propósito de esta investigación fue valorar qué factores son más determinantes con el grado de satisfacción de los trabajadores, así como identificar diferencias significativas en función del género, edad, nivel académico y área laboral. El estudio se llevó a cabo con una participación de 140 trabajadores del departamento de deportes de una Universidad Pública, de los cuales el $65.7 \%$ son hombres y el $34.3 \%$ son mujeres, siendo el mayor porcentaje de la muestra el grupo de edad que oscila entre los 26 a 35 con un $39.3 \%$. Los resultados fueron valorados satisfactoriamente por los trabajadores, respecto a los diferentes aspectos, como el lugar de trabajo, recursos materiales, seguridad e higiene, la organización del trabajo, reconocimiento y valoración global de su trabajo. Por otra parte, los resultados obtenidos arrojaron diferencias significativas únicamente en función de la edad $(p<.05)$. Para finalizar se calculó la fiabilidad de cada factor mediante el coeficiente alfa de Cronbach obteniendo valores que oscilan entre .76 y .89 .

Palabras claves: Satisfacción, trabajadores, organizaciones deportivas públicas, servicios deportivos. 
Revista de Investigación

Año 13.

Académica sin Frontera

Núm. 33

ISSN: 2007-8870

https://revistainvestigacionacademicasinfrontera.unison.mx/index.php/RDIASF/issue/view/22

Recibido el 23 de mayo de 2020. Dictaminado mediante arbitraje favorablemente 20 de diciembre de 2020.

\title{
Satisfaction of workers in a public educational organization that provides sports services.
}

\begin{abstract}
The satisfaction of the workers at present has taken special importance within the organizations, everything related to the worker, therefore it is considered as an indicator of quality within the management, being related to the efficiency of the services they offer. The purpose of this research was to assess which factors are most decisive with the degree of worker satisfaction, as well as identify significant differences based on gender, age, academic level and work area. The study was carried out with a participation of 140 workers from the sports department of a Public University, of which $65.7 \%$ are men and $34.3 \%$ are women, with the highest percentage of the sample being the age group that ranges from 26 to 35 with $39.3 \%$. The results were satisfactorily assessed by the workers, regarding the different aspects, such as the workplace, material resources, safety and hygiene, the organization of work, recognition and global assessment of their work. On the other hand, the results obtained showed significant differences only according to age $(p<.05)$. Finally, the reliability of each factor was calculated using Cronbach's alpha coefficient, obtaining values ranging between .76 and 89 .
\end{abstract}

Key Word: Satisfaction, workers, public sports organizations, sports services Introducción

La satisfacción laboral o el desempeño en las organizaciones toman especial relevancia, pues contribuyen de forma transversal a la gestión de $\mathrm{RR}$. $\mathrm{HH}$. y al bienestar de las personas, ya sea por medio de la detección de necesidades de capacitación, la formulación de programas de promoción y el diseño de sistemas de gestión del capital humano (Imran, Saeed, Anis-Ul-Haq \& Fatima, 2010). Un trabajador satisfecho, comprometido, que siente que sus aportaciones son tomadas en cuenta y que trabaja en equipo, tenderá a manifestar una mayor diligencia en el desempeño de su trabajo. Es por ello que los modelos de calidad total incluyen aspectos que inciden directamente en la gestión de los recursos humanos y, consecuentemente, en la satisfacción y el compromiso de los trabajadores (Mendoza, Orgambidez \& Carrasco, 2010).

La satisfacción del trabajador se relaciona directamente con la experiencia que vive dentro de la organización y después estas experiencias se transforman en la percepción del trabajador, y esta última culmina en un componente 


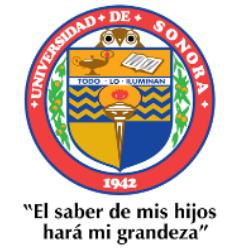

( Julio - Diciembre 2020)

Año 13.

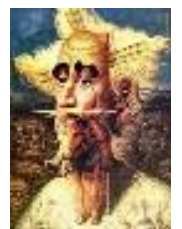

Revista de Investigación Académica sin Frontera ISSN: 2007-8870

https://revistainvestigacionacademicasinfrontera.unison.mx/index.php/RDIASF/issue/view/22

Recibido el 23 de mayo de 2020. Dictaminado mediante arbitraje favorablemente 20 de diciembre de 2020.

emocional, y después en la manera de actuar del individuo (Abrajan, Contreras \& Montoya, 2009). Tratando de delimitar y definir el concepto de satisfacción en el trabajo, muchas veces se encuentra con el hecho de que los diversos especialistas que han estudiado el tema, suelen utilizar indistintamente términos como el motivo del empleo, la actitud del trabajador, la moral laboral y la satisfacción en el trabajo, por lo que la satisfacción del trabajador se refiere a la orientación afectiva de las personas hacia los roles que desempeñan en su trabajo, si bien es cierto que podría adoptarse una concepción unitaria y generalista de satisfacción laboral, no se puede negar que se trata de una actitud global hacia el trabajo cuyas causas son multidimensionales (González, Sánchez \& López-Guzmán, 2011).

A pesar de las innumerables definiciones existentes acerca de satisfacción laboral González et al. (2011) argumentan que es posible categorizarlas a través de dos perspectivas distintas: la primera, como un estado emocional, un sentimiento o una respuesta afectiva hacia el trabajo; la segunda, como el resultado de una comparación entre expectativas o resultados actuales del puesto y las prestaciones que el trabajo ofrece realmente. Por ello las organizaciones, a la luz de los datos arrojados por la investigación, cada vez están más concienciadas con la importancia de disponer de trabajadores satisfechos con su trabajo y con la propia organización. Muchas tratan de lograr este objetivo interviniendo sobre las condiciones del contexto físico, aspectos tangibles o características de la tarea (Bòria-Reverter, Crespi-Vallbona \& Mascarilla-Miró, 2012).

Por lo anterior, el grado de satisfacción puede afectar la cantidad y calidad del trabajo que desempeñan los sujetos dentro de sus puestos de trabajos, por lo que la satisfacción laboral es un clásico indicador al que se ocurre cuando se desea conocer la actitud general de las personas dentro de las organizaciones (González, Guevara, Morales, Segura \& Luengo. 2013). Por lo que el propósito de las organizaciones es crecer, lo que conlleva a conocer a las personas que laboran dentro de ella, lo que implica valorar el capital humano y para ello es importante saber el punto de vista del trabajador y el grado de satisfacción laboral que experimentan (Abrajan et al., 2009).

Para reforzar esta investigación, se procedió a la revisión de literatura científica, encontrando diversas investigaciones que han utilizado instrumentos de medición para estudiar la satisfacción del trabajador, así como de todos esos aspectos que generan satisfacción (Jun \& Cai, 2010). Encontrando autores que 
Revista de Investigación

Año 13.

Académica sin Frontera

Núm. 33

ISSN: 2007-8870

https://revistainvestigacionacademicasinfrontera.unison.mx/index.php/RDIASF/issue/view/22

Recibido el 23 de mayo de 2020. Dictaminado mediante arbitraje favorablemente 20 de diciembre de 2020.

estudian de manera conjunta las características personales y laborales. (Ayestarán \& Valencia, 2010; Cohrs, Abele, \& Dette, 2006; Franek \& Večeřa, 2008; Gazioglu \& Tansel, 2006. Según expresa Gamero (2003) las características personales como la edad, género, nivel educativo, entre otras, se consideran influyentes en la satisfacción laboral. Otros estudios se han enfocado en explorar las complejas relaciones entre la motivación (Stringer, Didham \& Theivananthampillai, 2011), la influencia de las características demográficas, especialmente en género (Belias \& Koustelios, 2014; Belias, Koustelios, Sdrollias \& Koutiva, 2013). Las medidas de remuneración y satisfacción laboral de pago (Judge, Piccolo, Podsakoff, Shaw \& Rich, 2010; Lane, Esser, Holte \& McCusker, 2010). La relación con los compañeros, crecimiento, las condiciones físicas y la percepción de la cultura específica de su organización (Antoncic \& Antoncic, 2011; Pelit, Öztürk \& Arslantürk, 2011).

\section{Método}

\section{Tipo de investigación}

Se realizó un estudio cuantitativo y descriptivo mediante la utilización de encuesta, de carácter transversal por su aplicación en un único memento temporal y no experimental (Hernández, Fernández \& Baptista, 2014).

\section{Participantes}

Los participantes fueron $n=140$ trabajadores del departamento de deportes dentro una universidad pública en Obregón, Sonora, México, siendo el $65.7 \%$ hombres y el $34.3 \%$ mujeres, siendo el mayor porcentaje de la muestra el grupo de edad que oscila entre los 26 a 35 con un 39.3\% y el menor grupo de personas refleja que son > 56 años con un 3.6\%. En cuanto al nivel de estudios el $55.7 \%$ de los trabajadores cuenta con grado de licenciatura, según el área laboral, resaltando los instructores/entrenadores con un $40.7 \%$ (ver tabla 1 y 2).

Tabla 1

Información personal del trabajador

\begin{tabular}{llcc}
\hline \multicolumn{1}{c}{ Variable } & \multicolumn{1}{c}{ Descripción } & F & $\%$ \\
\hline \multirow{2}{*}{ Género } & Hombre & 92 & 65.7 \\
& M ujer & 48 & 34.3 \\
\multirow{5}{*}{ Edad } & De 18 a 25 años & 44 & 31.4 \\
& De 26 a 35 años & 55 & 39.3 \\
& De 26 a 35 años & 21 & 15.0 \\
& De 46 a 55 años & 15 & 10.7 \\
& > de 56 años & 5 & 3.6 \\
\multirow{5}{*}{ Nivel de estudios } & Primaria & 3 & 2.1 \\
& Secundaria & 7 & 5.0 \\
& Preparatoria & 16 & 11.4 \\
& Técnica & 9 & 6.4 \\
& Licenciatura & 78 & 55.7 \\
Nota: $:$ F = Frecuanica, \% = Porcentaje & 27 & 19.3 \\
\hline & Posgrado & &
\end{tabular}

Tabla 2

Estadisticos descriptivos área/puesto de trabajo

\begin{tabular}{lcc}
\hline \multicolumn{1}{c}{ Descripción } & $\mathrm{F}$ & $\%$ \\
\hline Instructor/Entrenador & 57 & 40.7 \\
Área Metodológica & 10 & 7.1 \\
Área Administrativa & 12 & 8.6 \\
Área de Dirección & 6 & 4.3 \\
Área de Vigilanacia & 10 & 7.1 \\
Área de Mantenimiento e Intendencia & 10 & 7.1 \\
Área de almacén & 5 & 3.6 \\
Ppto. de Deporte y Salud & 6 & 4.3 \\
Extensión Deportiva & 24 & 17.1 \\
\hline Nota: F = Frecuanica, \% = Porcentaje & &
\end{tabular}


Revista de Investigación

Año 13.

Académica sin Frontera

Núm. 33

ISSN: 2007-8870

https://revistainvestigacionacademicasinfrontera.unison.mx/index.php/RDIASF/issue/view/22

Recibido el 23 de mayo de 2020. Dictaminado mediante arbitraje favorablemente 20 de diciembre de 2020.

\section{Instrumento.}

Se utilizó la encuesta de satisfacción del trabajador de Marqués (2002), adaptado al contexto mexicano por Reyes-Robles, Medina-Rodríguez, RamírezSiqueiros, López-Walle, y Ceballos-Gurrola (2018), mencionando que el proceso se llevó a cabo con la ayuda de expertos en la materia. Está conformado por 28 ítems, la escala para valorar cada ítem, se optó por una tipo Likert, con valores de 1 a 4 , donde 1 = Nada satisfecho. 2 = Poco. 3 = Algo. 4 = Muy satisfecho. Cada uno de ellas está teóricamente definida de la siguiente manera:

Identificación personal: en este apartado se trata de conocer el perfil básico de cada trabajador, sobre su edad, género, estudios realizados y área/departamento al que pertenece.

Los medios físicos para el desempeño de su trabajo: en este apartado se trata de saber en qué medida le resulta satisfactorio el lugar donde desempeña su trabajo, los recursos materiales de los que dispone y el grado de seguridad e higiene de su puesto de trabajo, que incluye los ítems del 1 al 12.

La organización del trabajo: este apartado se utilizara para conocer qué opinión le merece la estructura del trabajo que realiza, si están claras sus funciones y responsabilidades, las posibilidades de aportar mejoras, que incluye los ítems del 13 al 19.

El reconocimiento del trabajo: fue diseñado para saber cómo valora la organización el trabajo desempeñado por él y si consideraba que estuviera debidamente retribuido en relación con otras organizaciones similares, que incluye los ítems del 20 al 21.

Una valoración global del trabajo que desempeña: en este apartado se pregunta en qué medida se está satisfecho con diversos aspectos como el compromiso con los objetivos, la capacidad de adaptación de la organización a los intereses personales, que incluye los ítems del 22 al 28.

\section{Procedimiento.}

Con previa autorización, un grupo de encuestadores visitaron las áreas de trabajo, allí se explicó el objetivo del estudio, se describió la encuesta y la forma de llenado, cabe señalar que en todo momento se aseguró el anonimato y la confidencialización de los datos recogidos. Participando así de forma voluntaria 
Revista de Investigación

Año 13.

Académica sin Frontera

Núm. 33

ISSN: 2007-8870

https://revistainvestigacionacademicasinfrontera.unison.mx/index.php/RDIASF/issue/view/22

Recibido el 23 de mayo de 2020. Dictaminado mediante arbitraje favorablemente 20 de diciembre de 2020.

cada trabajador, el cuestionario fue auto administrado, lo que significa que se proporcionó directamente a los participantes, quienes los contestaron.

\section{Análisis de datos.}

Una vez tabulados los datos, estos fueron analizados mediante el paquete estadístico SPSS v24, llevando a cabo como proceso inicial la depuración y calidad de los datos, segundo, se obtuvieron estadísticos descriptivos sobre la valoración respecto al grado de satisfacción a cada uno de los aspectos evaluados, después, se llevó a cabo análisis inferencial para constatar estadísticamente la existencia de diferencia de medias en las valoraciones, para ello se identificó si la variable cuantitativa analizada seguía una distribución normal. La prueba de Kolmogorov-Smirnov $(K-S)$ refleja que todos los valores del estadístico $p<.01$, por lo que las variables analizadas siguen una distribución no normal. Por ello, se comprobó la diferencia de medias a través de pruebas no paramétricas de $U$ de Mann-Whitney, y Kruskal-Wallis. Para finalizar se determinó la fiabilidad de cada uno de los factores de la escala, la cual fue calculada mediante el índice alfa de Cronbach (Cronbach, 1951).

\section{Resultados}

Al analizar en primer lugar los aspectos relacionados al lugar de trabajo que la entidad le ha asignado al trabajador para el funcionamiento de su trabajo en cuanto a disponer y adecuar un espacio acorde a las necesidades propias del área. Se observa que la población de trabajadores representada por un $57 \%$ se halla muy satisfechos con los elementos de adecuación, facilidad y cantidad de espacio proporcionada en su área de trabajo (ver figura 1).

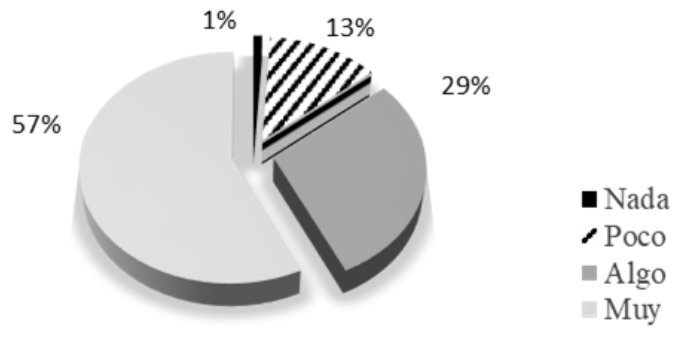

Figura 1. Valoración sobre la satisfacción del lugar de trabajo (espacio de trabajo). 
"El saber de mis hijos

hará mi grandeza"

Año 13.

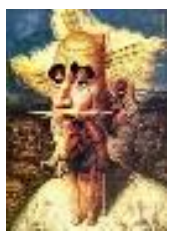

Revista de Investigación

Académica sin Frontera

ISSN: 2007-8870

https://revistainvestigacionacademicasinfrontera.unison.mx/index.php/RDIASF/issue/view/22

Recibido el 23 de mayo de 2020. Dictaminado mediante arbitraje favorablemente 20 de diciembre de 2020.

En lo tocante a la cantidad de recursos materiales que la entidad deportiva proporciona a los trabajadores como insumos y herramientas para disponer para la realización del trabajo. Los resultados muestran que la población de trabajadores se encuentra algo satisfechos representada en un $47 \%$. Tomando en cuenta la valoración de los elementos en cuanto a la facilidad que tienen para disponer de los recursos materiales y al mismo tiempo que los mismos no son adecuados ni suficientes (ver figura 2).

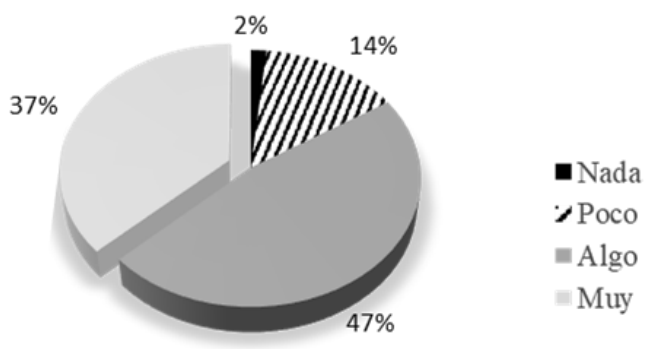

Figura 2. Valoración sobre la satisfacción de los recursos materiales.

En lo referente al factor seguridad e higiene del entorno de su trabajo, tomando en cuenta que es uno de los aspectos que suelen tomar mucha importancia por el personal de la entidad, considerando las normativas establecidas, los espacios seguros y un entorno medioambiental acorde, además de la funcionalidad y ergonomía que logran en conjunto una mayor productividad en el trabajador. Los resultados muestran que un $45 \%$ de la población de trabajadores se encuentra muy satisfecha, un $48 \%$ está algo satisfecha (ver figura 3).

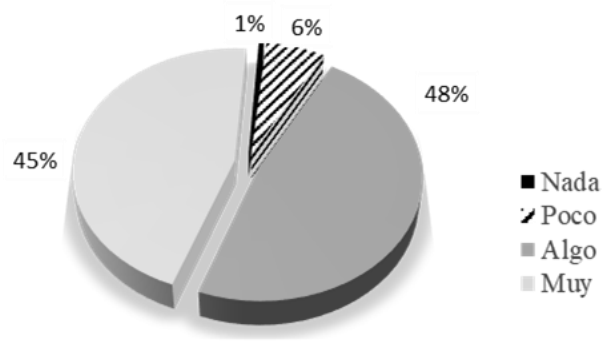

Figura 3. Valoración sobre la satisfacción con la seguridad e higiene dentro de su trabajo. 
"El saber de mis hijos

hará mi grandeza"

Año 13.

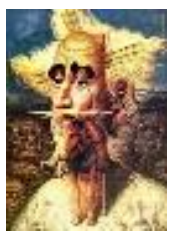

Revista de Investigación

Académica sin Frontera

ISSN: 2007-8870

https://revistainvestigacionacademicasinfrontera.unison.mx/index.php/RDIASF/issue/view/22

Recibido el 23 de mayo de 2020. Dictaminado mediante arbitraje favorablemente 20 de diciembre de 2020.

Para el trabajador los aspectos relacionados a la organización del trabajo enfocado a la delimitación de funciones, apertura a aportar propuestas para lograr la eficiencia, el otorgamiento de responsabilidades para la información y comunicación fluida, así como los horarios establecidos en todos los niveles de la organización, entre otros. Los resultados muestran que el $68 \%$ de la población se halla muy satisfecha, el $29 \%$ algo satisfechos (ver figura 4 ).

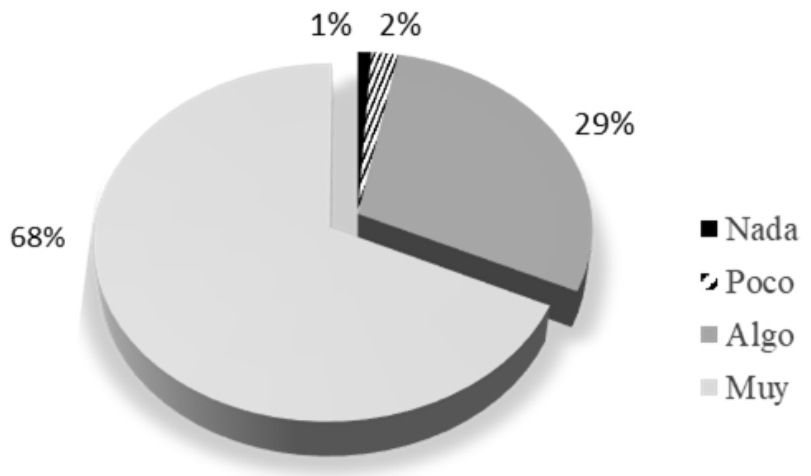

Figura 4. Valoración sobre la satisfacción en la organización del trabajo

En lo tocante al factor reconocimiento que otorga o recibe del desempeño de manera intrínseca o con incentivos otorgados para el logro de metas personales dentro de la entidad, se observa que la población de los trabajadores representada por un $33 \%$ se halla poco satisfecha, y un $28 \%$ manifiesta estar algo satisfecho con la retribución económica que recibe respecto a puestos similares al suyo en otras organizaciones, así como las posibilidades de formación como acreditaciones, certificaciones, capacitación, entre otras (ver figura 5).

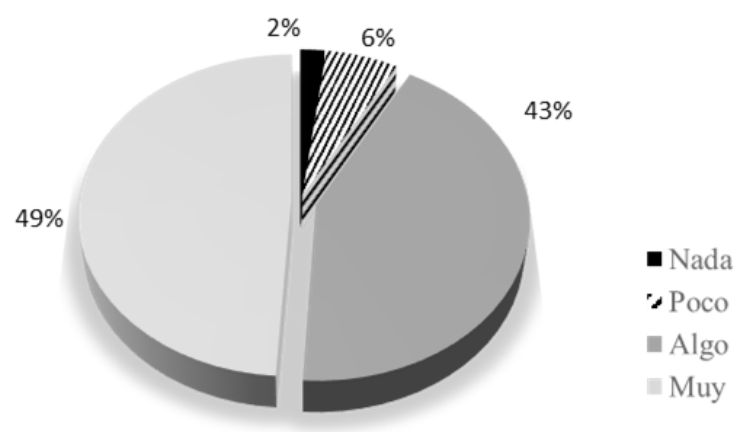

Figura 5. Valoración sobre la satisfacción con el reconocimiento sobre el desempeño de su trabajo 
"El saber de mis hijos

hará mi grandeza"

Año 13.

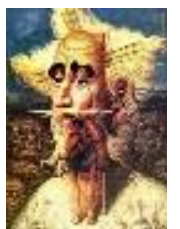

Revista de Investigación

Académica sin Frontera

ISSN: 2007-8870

https://revistainvestigacionacademicasinfrontera.unison.mx/index.php/RDIASF/issue/view/22

Recibido el 23 de mayo de 2020. Dictaminado mediante arbitraje favorablemente 20 de diciembre de 2020.

Para finalizar, se analiza lo referente a todos los aspectos relacionados con la valoración global del trabajo para lograr los objetivos y el compromiso que adquiere hacia la organización para dicho cumplimiento. Los resultados arrojan que la población de trabajadores esta algo satisfecha representada por un $23 \%$ y un $74 \%$ manifiesta estar muy satisfecha. Mientras que un número pequeño de trabajadores representada por un $3 \%$ manifiesta no estar satisfecho con la disposición para adaptarse a sus necesidades e intereses en el desempeño de su trabajo, compromiso con el objetivo de la entidad, con el trabajo que desempeña, disposición para atender sus necesidades e intereses personales, con el ambiente de trabajo, así como las expectativas personales no están satisfechas con el trabajo que realiza (ver figura 6).

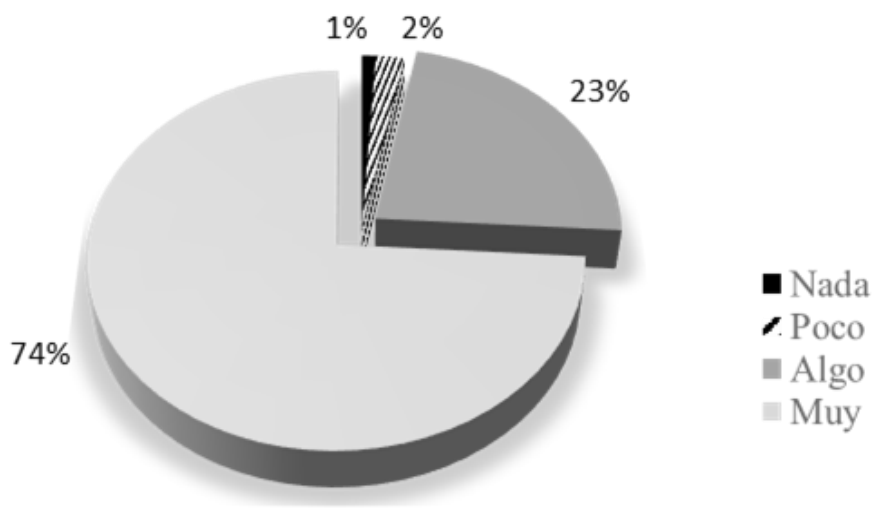

Figura 6. Satisfacción con la valoracion global de su trabajo

Al comparar las medias de satisfacción del trabajador con cada uno de los factores en función del género, no se encontraron diferencias estadísticamente significativas $(p>.05)$. No obstante, cabe mencionar que los hombres valoraron mejor lo relacionado al lugar de trabajo, recursos materiales, seguridad e higiene y reconocimiento, mientras que las mujeres manifestaron estar mejor con la organización en el trabajo y valoración global (ver tabla 3). 
"El saber de mis hijos

hará mi grandeza"

Año 13.

Núm. 33
Revista de Investigación

Académica sin Frontera

ISSN: 2007-8870

https://revistainvestigacionacademicasinfrontera.unison.mx/index.php/RDIASF/issue/view/22

Recibido el 23 de mayo de 2020. Dictaminado mediante arbitraje favorablemente 20 de diciembre de 2020.

Tabla 3

Satisfacción del trabajador en función del género

\begin{tabular}{lccccc}
\hline \multirow{2}{*}{ Factores } & \multicolumn{2}{c}{$\begin{array}{c}\text { Hombre } \\
(\mathrm{N}=92)\end{array}$} & \multicolumn{2}{c}{$\begin{array}{c}\text { Mujer } \\
(\mathrm{N}=48)\end{array}$} & \\
\cline { 2 - 5 } & $M$ & $D T$ & $M$ & $D T$ & \\
\cline { 2 - 5 } & 3.30 & .62 & 3.22 & .61 & .441 \\
Lugar de trabajo & 3.08 & .69 & 3.03 & .67 & .683 \\
Recursos materiales & 3.27 & .57 & 3.25 & .52 & .697 \\
Seguridad e higiene & 3.31 & .49 & 3.35 & .51 & .647 \\
Organización en el & 3.19 & .70 & 3.10 & .71 & .636 \\
trabajo & 3.35 & .47 & 3.43 & .44 & .397 \\
Reconocimiento & & & & &
\end{tabular}

Tras agrupar las diferentes edades de los trabajadores en cinco grupo, sólo se encontraron diferencias estadísticamente significativas en el factor de reconocimiento $(p<.05)$, donde el grupo 18 a 25 años de edad percibió mayores niveles de satisfacción que el resto de los grupos de edad (ver tabla 4)

Tabla 4

Satisfacción del trabajador en función del grupo de edad

\begin{tabular}{|c|c|c|c|c|c|c|c|}
\hline Factores & $\begin{array}{c}\text { De } 18 \text { a } \\
25\end{array}$ & $\begin{array}{c}\text { De } 26 \text { a } \\
35\end{array}$ & $\begin{array}{c}\text { De } 36 \text { a } \\
45\end{array}$ & $\begin{array}{c}\text { De } 46 \text { a } \\
55\end{array}$ & $>$ de 56 & Chi $^{2}$ & $P$ \\
\hline $\begin{array}{l}\text { Lugar de } \\
\text { trabajo }\end{array}$ & $3.32 \pm .62$ & $\begin{array}{c}3.20 \pm .5 \\
4\end{array}$ & $\begin{array}{c}3.38 \pm .6 \\
4\end{array}$ & $\begin{array}{c}3.22 \pm .8 \\
5\end{array}$ & $\begin{array}{c}3.26 \pm .4 \\
3\end{array}$ & 3.215 & $\begin{array}{c}.52 \\
3\end{array}$ \\
\hline $\begin{array}{l}\text { Recursos } \\
\text { materiales }\end{array}$ & $3.21 \pm .64$ & $\begin{array}{c}2.93 \pm .6 \\
7\end{array}$ & $\begin{array}{c}3.01 \pm .7 \\
0\end{array}$ & $\begin{array}{c}3.02 \pm .8 \\
0\end{array}$ & $\begin{array}{c}3.46 \pm .5 \\
0\end{array}$ & 6.048 & $\begin{array}{c}.19 \\
6\end{array}$ \\
\hline $\begin{array}{l}\text { Seguridad e } \\
\text { higiene }\end{array}$ & $3.34 \pm .52$ & $\begin{array}{c}3.15 \pm .5 \\
8\end{array}$ & $\begin{array}{c}3.26 \pm .5 \\
1\end{array}$ & $\begin{array}{c}3.38 \pm .5 \\
8\end{array}$ & $\begin{array}{c}3.43 \pm .3 \\
8\end{array}$ & 4.552 & $\begin{array}{c}.33 \\
6\end{array}$ \\
\hline $\begin{array}{l}\text { Organización } \\
\text { en el trabajo }\end{array}$ & $3.43 \pm .47$ & $\begin{array}{c}3.28 \pm .4 \\
9\end{array}$ & $\begin{array}{c}3.23 \pm .5 \\
3\end{array}$ & $\begin{array}{c}3.23 \pm .5 \\
3\end{array}$ & $\begin{array}{c}3.54 \pm .2 \\
7\end{array}$ & 3.769 & $\begin{array}{c}.43 \\
8\end{array}$ \\
\hline Reconocimient & $3.40 \pm .64$ & $3.01 \pm .7$ & $3.05 \pm .6$ & $\begin{array}{c}3.16 \pm .6 \\
7\end{array}$ & 3.30土.7 & 10.15 & .03 \\
\hline $\begin{array}{l}\text { Valoración } \\
\text { global }\end{array}$ & $3.47 \pm .38$ & $\begin{array}{c}3.35 \pm .4 \\
7\end{array}$ & $3.19 \pm 53$ & $\begin{array}{c}3.43 \pm .4 \\
4\end{array}$ & $\begin{array}{c}3.40 \pm .5 \\
1\end{array}$ & 4.595 & $\begin{array}{c}.33 \\
1\end{array}$ \\
\hline
\end{tabular}


https://revistainvestigacionacademicasinfrontera.unison.mx/index.php/RDIASF/issue/view/22

ISSN: 2007-8870

Recibido el 23 de mayo de 2020. Dictaminado mediante arbitraje favorablemente 20 de diciembre de 2020.

Al analizar la satisfacción de los trabajadores en función del nivel académico no se hallaron diferencias estadísticamente significativas $(p>.05)$, no obstante los trabajadores con nivel de preparatoria valoraron mejor los factores lugar de trabajo y reconocimiento, mientras que el grupo con nivel técnico valoraron mejor los recursos materiales, seguridad e higiene y la organización en el trabajo y por último el grupo de secundaria valoro mejor la valoración global (ver tabla 5)

Tabla 5

Satisfacción del trabajador en función del nivel de estudios

\begin{tabular}{|c|c|c|c|c|c|c|c|c|}
\hline Factores & $\begin{array}{c}\text { Primar } \\
\text { ia }\end{array}$ & $\begin{array}{l}\text { Secund } \\
\text { aria }\end{array}$ & $\begin{array}{c}\text { Preparat } \\
\text { oria }\end{array}$ & $\begin{array}{l}\text { Técnic } \\
\text { a }\end{array}$ & $\begin{array}{c}\text { Licenciat } \\
\text { ura }\end{array}$ & $\begin{array}{c}\text { Posgra } \\
\text { do }\end{array}$ & $\mathrm{Chi}^{2}$ & $P$ \\
\hline $\begin{array}{l}\text { Lugar de } \\
\text { trabajo }\end{array}$ & $\begin{array}{c}2.88 \pm . \\
83\end{array}$ & $\begin{array}{c}3.14 \pm .4 \\
2\end{array}$ & $3.54 \pm .52$ & $\begin{array}{c}3.51 \pm . \\
41\end{array}$ & $3.20 \pm .65$ & $\begin{array}{c}3.29 \pm . \\
58\end{array}$ & $\begin{array}{l}7.1 \\
37\end{array}$ & $\begin{array}{c}.21 \\
1\end{array}$ \\
\hline $\begin{array}{l}\text { sos } \\
\text { iales }\end{array}$ & $\begin{array}{c}2.77 \pm . \\
50\end{array}$ & $\begin{array}{c}3.28 \pm .5 \\
9\end{array}$ & $3.18 \pm .53$ & $\begin{array}{c}3.29 \pm . \\
48\end{array}$ & $3.02 \pm .72$ & $\begin{array}{c}3.00 \pm . \\
72\end{array}$ & $\begin{array}{l}3.1 \\
85\end{array}$ & $\begin{array}{c}.67 \\
2\end{array}$ \\
\hline $\begin{array}{l}\text { idad e } \\
\text { le }\end{array}$ & $\begin{array}{c}3.00 \pm . \\
88\end{array}$ & $\begin{array}{c}2.30 \pm .5 \\
8\end{array}$ & $3.41 \pm .45$ & $\begin{array}{c}3.46 \pm . \\
50\end{array}$ & $3.24 \pm .55$ & $\begin{array}{c}3.17 \pm . \\
55\end{array}$ & $\begin{array}{l}3.8 \\
49\end{array}$ & $\begin{array}{c}.57 \\
1\end{array}$ \\
\hline $\begin{array}{l}\text { Organizació } \\
\text { n en el } \\
\text { trabajo }\end{array}$ & $\begin{array}{c}3.28 \pm . \\
65\end{array}$ & $\begin{array}{c}3.42 \pm .6 \\
1\end{array}$ & $3.38 \pm .49$ & $\begin{array}{c}3.50 \pm . \\
45\end{array}$ & $3.31 \pm .48$ & $\begin{array}{c}3.23 \pm . \\
51\end{array}$ & $\begin{array}{l}1.7 \\
37\end{array}$ & $\begin{array}{c}.88 \\
4\end{array}$ \\
\hline Reconocimi & $\begin{array}{c}3.33 \pm . \\
57\end{array}$ & $\begin{array}{c}3.14 \pm .8 \\
5\end{array}$ & $3.34 \pm .56$ & $\begin{array}{c}3.16 \pm . \\
43\end{array}$ & $3.19 \pm .73$ & $\begin{array}{c}3.07 \pm . \\
68\end{array}$ & $\begin{array}{l}4.1 \\
23\end{array}$ & $\begin{array}{c}.53 \\
2\end{array}$ \\
\hline $\begin{array}{l}\text { Valoración } \\
\text { global }\end{array}$ & $\begin{array}{c}3.00 \pm . \\
49\end{array}$ & $\begin{array}{c}3.46 \pm .5 \\
3\end{array}$ & $3.38 \pm .48$ & $\begin{array}{c}3.44 \pm . \\
44\end{array}$ & $3.39 \pm .41$ & $\begin{array}{c}3.33 \pm . \\
55\end{array}$ & $\begin{array}{l}3.0 \\
91\end{array}$ & $\begin{array}{l}.68 \\
6\end{array}$ \\
\hline
\end{tabular}

Por otro lado al comparar las áreas o departamentos laborales no se hallaron diferencias estadísticamente significativas $(p>.05)$, no obstante las valoraciones más bajas las obtuvo el factor de recursos materiales. Por otra parte, el área o departamento de Deporte y Salud fue el que obtuvo las valoraciones más bajas al resto de las áreas y los departamentos de Dirección (deportes) y Extensión Deportiva otorgaron las valoraciones más altas a cada uno de los aspectos evaluados, haciendo énfasis al aspecto de reconocimiento. 
Revista de Investigación

Año 13.

Académica sin Frontera

Núm. 33

ISSN: 2007-8870

https://revistainvestigacionacademicasinfrontera.unison.mx/index.php/RDIASF/issue/view/22

Recibido el 23 de mayo de 2020. Dictaminado mediante arbitraje favorablemente 20 de diciembre de 2020.

\section{Análisis de fiabilidad}

La consistencia interna fue calculada mediante el índice alfa de Cronbach (Cronbach, 1951), en lo que se refiere a los factores que componen el instrumento, el valor más bajo corresponde al factor de reconocimiento con un índice de .77 y el factor de seguridad y recursos materiales ha obtenido un índice de .85 (ver tabla 6$)$.

Tabla 6

Fiabilidad de consistencia interna por factor

\begin{tabular}{lcc}
\hline Factor & № Items & $\alpha$ \\
\hline Lugar de trabajo & 3 & .80 \\
Recursos materiales & 3 & .83 \\
Seguridad e Higiene & 6 & .85 \\
Organización del trabajo & 5 & .81 \\
Reconocimiento & 5 & .77 \\
Valoración global de su trabajo & 6 & .79 \\
\hline
\end{tabular}

Nota: $\alpha=$ alfa de Cronbach

\section{Discusión}

El propósito de esta investigación fue valorar qué factores son más determinantes con el grado de satisfacción de los trabajadores, así como identificar diferencias significativas en función del género, edad, nivel académico y área laboral. En este sentido se analizaron estos factores de acuerdo a los resultados obtenidos en la presente investigación con otros estudios realizados.

Al ser analizadas los factores obtenidos en este estudio, los resultados fueron valorados satisfactoriamente por los trabajadores, esto indica que existe buena satisfacción en cada una de las áreas o departamentos laborales, abarcando diferentes aspectos, como el lugar de trabajo, recursos materiales, seguridad e higiene, la organización del trabajo, reconocimiento y valoración global de su trabajo (Reyes, 2018). Coincidiendo con otro estudio indicando que la satisfacción general por los trabajadores, es media-alta (Bòria-Reverter, 


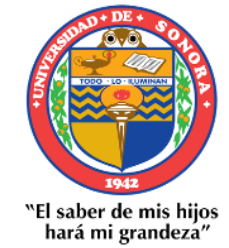

( Julio - Diciembre 2020)

Año 13.

Núm. 33

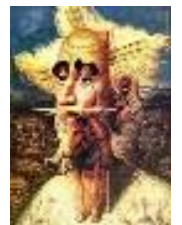

Revista de Investigación

Académica sin Frontera

ISSN: 2007-8870

https://revistainvestigacionacademicasinfrontera.unison.mx/index.php/RDIASF/issue/view/22

Recibido el 23 de mayo de 2020. Dictaminado mediante arbitraje favorablemente 20 de diciembre de 2020.

2012). No obstante en otros estudios, en los resultados encontrados se manifiesta que la población estudiada está moderadamente satisfecha (Medina, Ceballos, Giner y Marqués; 2009; Rodríguez, 2016).

Al comparar las medias de satisfacción del trabajador con cada uno de los factores en función del género, no podemos decir que los hombres tienen satisfacción laboral diferente a la de las mujeres, coincidiendo con el estudio de (Aznar, 2015; Reyes, 2018). Por otra parte, en otros estudios si se encontraron diferencias significativas $(p<.05)$ arrojando que la satisfacción de los hombres difiere a las mujeres (Belias, et al; 2013; Jung, Monn, Hahm, 2007; Medina et al., 2009), mientras que en otro estudio por Gamero (2003) menciona que las mujeres estas más satisfechas que los hombres.

Por otra parte, se encontraron diferencias estadísticamente significativas en función de la edad, donde los jóvenes de 18 a 25 años difieren con el grupo de edad entre 26 a 35 años de edad $(p<.05)$, coincidiendo nuestros resultados con otros estudios que si hallaron diferencias, sólo que en trabajadores mayores a 45 años de edad respecto a los trabajadores jóvenes (Belias, et al., 2013; Bernabé, Campos-Izquierda \& González, 2016; Choudhury \& Gupta, 2011; Belias, Koustelios, Sdrollias, Koutiva \& Zournatzi, 2013). Por otra parte, al analizar la satisfacción de los trabajadores en función del nivel académico, en nuestro estudio no se hallaron diferencias significativas $(p>.05)$ coincidiendo así, con los resultados de Medina et al. (2009). A diferencia de otros estudios, manifestando que los trabajadores que tienen hasta un nivel de secundaria y preparatoria difieren del grupo de trabajadores que cuentan con nivel de licenciatura (Bader, Hashim \& Zaharim, 2013; Belias et al., 2013; Reyes, 2018).

Por otro lado al comparar las áreas o departamentos laborales no se hallaron diferencias estadísticamente significativas $(p>.05)$, Resultado que coincide con el estudio de Bader et al. (2013) mostrando que tanto los gerentes de alto nivel, de departamento, así como el personal en general experimentan aproximadamente los mismos niveles de satisfacción laboral. No obstante, en nuestro estudio el personal del área de dirección (deportes) otorga valoraciones más altas al factor reconocimiento, hallazgo que concuerda con Medina et al. (2009) que los directivos son los que están más satisfechos que el resto de las áreas. Por otro lado, Belias et al. (2013) mencionan que los empleados que ocupan puestos más altos en una institución son más propensos a creer que su ambiente de trabajo es agradable, que su salario es el adecuado para cubrir sus necesidades. 
Revista de Investigación

Año 13.

Académica sin Frontera

Núm. 33

ISSN: 2007-8870

https://revistainvestigacionacademicasinfrontera.unison.mx/index.php/RDIASF/issue/view/22

Recibido el 23 de mayo de 2020. Dictaminado mediante arbitraje favorablemente 20 de diciembre de 2020.

Por último comprobó la fiabilidad del instrumento mediante análisis de consistencia interna, determinándose el coeficiente alfa de Cronbach (Cronbach, 1951) para cada factor, obteniendo como el valor más bajo correspondiente al factor 5 con un índice de .77, y el valor más alto corresponde al factor 3 con un índice de .85, teniendo en cuenta el rango establecido por Oviedo y CamposArías (2005) para aceptar la consistencia interna, se afirma que los resultados obtenidos son buenos considerando que existe homogeneidad entre los ítems dentro de cada factor (Kaplan \& Saccuzzo, 2006).

\section{Conclusión}

De acuerdo a los resultados obtenidos, el estudio ha permitido conocer el grado de satisfacción de los trabajadores del departamento de deportes dentro una universidad pública en Obregón, Sonora, México, por lo que este este tipo de estudios son de vital importancia para conocer la opinión que tienen los trabajadores, y percepciones, lo cual permite a los gestores tomar decisiones que permitan adaptar mejor los necesidades de sus trabajadores, y de este modo incidir de forma positiva en las satisfacción de los mismos, acreditando también como útil para la gestión y la investigación, pudiendo ser utilizado por los responsables de las organizaciones de forma periódica. Como futura propuesta, se recomienda aplicar el instrumento a poblaciones más amplias para valorar mejor su utilidad.

\section{Referencias bibliográfica}

1. Abrajan, M., Contreras, J. \& Montoya, S. (2009). Grado de satisfacción laboral y condiciones de trabajo: una exploración cualitativa. Enseñanza e investigación en Psicología, 14(1), 105-118.

2. Antoncic, J., \& Antoncic, B. (2011). Employee satisfaction, intrapreneurship and firm growth: a model. Industrial Management \& Data Systems, 111(4), 589-607.

3. Ayestarán, S. y Valencia, J. (2010). ¿Cómo influyen los cambios culturales sobre el contrato psicológico entre el trabajador y la organización? Revista de Psicología Social, 25(2), 131-144.

4. Aznar, M. (2015). Análisis de las actividades físicas y deportivas extraescolares en los centros de enseñanza secundaria de la ciudad de Zaragoza (Tesis doctoral). Universidad Pablo Olavide, Sevilla. 
Año 13.

Núm. 33

https://revistainvestigacionacademicasinfrontera.unison.mx/index.php/RDIASF/issue/view/22
Revista de Investigación

Académica sin Frontera

ISSN: 2007-8870

Recibido el 23 de mayo de 2020. Dictaminado mediante arbitraje favorablemente 20 de diciembre de 2020.

5. Bader, H., Hashim, I., \& Zaharim, N. (2013). Job Satisfaction among Bank Employees in Eastern Libya. American International Journal of Social Science, 2(1), 30-44

6. Belias, D., \& Koustelios, A. (2014). Transformational Leadership and Job Satisfaction in the Banking Sector: A Review. International Review of Management and Marketing, 4(3), 187-200.

7. Belias, D., Koustelios, A., Sdrollias, L., \& Koutiva, M. (2013). The influence of demographic features on the job satisfaction of Greek bank employees. International Journal of Human Resource Management and Research, 3(4), 15-28.

8. Bernabé, B., Campos-Izquierdo, A., y González, M. (2016). Job satisfaction of sport and physical activity instructors in Spain according to gender and age. South African Journal for Research in Sport, Physical Education and Recreation, 38(1) 1-12.

9. Bòria-Reverter, S., Crespi-Vallbona, M., \& Mascarilla-Miró, O. (2012). Variables determinantes de la satisfacción laboral en España. Cuadernos de economía, 35(97), 9-16.

10. Cronbach, L. J. (1951). Coefficient alpha and the internal structure of tests. Psychometrika, 16, 1-16

11. Cohrs, J., Abele, A., y Dette, D. (2006). Integrating situational and dispositional determinants of job satisfaction: findings from three samples of professionals. The Journal of Psychology, 140(4), 363-395. doi: 10.3200/JRLP.140.4.363-395

12. Choudhury, R., \& Gupta, V. (2011). Impact of Age on Pay Satisfaction and Job Satisfaction leading to Turnover Intention: A Study of Young Working Professionals in India. Management and Labour Studies, 36(4), 353-363.

13. Franek, M. \& Večeřa, J. (2008). Personal characteristics and job satisfaction. E+M Ekonomie a Management, 4, 63-76.

14. Gamero, C. (2003). Análisis Económico de la Satisfacción Laboral. (Tesis doctoral). España. Universidad de Málaga.

15. Gazioglu, S., y Tansel, A. (2006). Job satisfaction in Britain: individual and job related factors. Applied Economics, 38(10), 1163-1171.

16. González, F., Sánchez, S., \& López-Guzmán, T. (2011). Satisfacción laboral como factor crítico para la calidad: el caso del sector hostelero de la provincia de Córdoba-España. Estudios y Perspectivas en Turismo, 20, 1047-1068.

17. González, L., Guevara, E., Morales, G., Segura, P., \& Luengo, C. (2013). Relación de la satisfacción laboral con estilos de liderazgo en enfermeros de hospitales públicos. Ciencia y enfermería, 19(1), 11-21. 
Revista de Investigación

Año 13.

Académica sin Frontera

Núm. 33

ISSN: 2007-8870

https://revistainvestigacionacademicasinfrontera.unison.mx/index.php/RDIASF/issue/view/22

Recibido el 23 de mayo de 2020. Dictaminado mediante arbitraje favorablemente 20 de diciembre de 2020.

18. Hernández, R., Fernández, C. \& Baptista, P. (2014). Metodología de la investigación. (6 ed.). México: McGraw-Hill.

19. Imran, R., Saeed, T., Anis-UI-Haq, M., \& Fatima, A. (2010). Organizational climate as a predictor of innovative work behavior. African Journal of Business Management, 15, 3337-3343.

20. Judge, T., Piccolo, R., Podsakoff, N., Shaw, J., \& Rich, B. (2010). The relationship between pay and job satisfaction: $A$ meta-analysis of the literature. Journal of Vocational Behavior, 77(2), 157-167. doi:10.1016/j.jvb.2010.04.002

21. Jun, M., \& Cai, S. (2010). Examining the relationships between internal service quality and its dimensions, and internal customer satisfaction. Total Quality Management, 21(2), 205-223. doi: 10.1080/14783360903550095

22. Jung, K., Moon, M., \& Hahm, S. (2007). Do age, gender, and sector affect job satisfaction? Results from the Korean labor and income panel data. Review of Public Personnel Administration, 27(2), 125-146.

23. Kaplan, R., \& Saccuzzo, D. (2006). Pruebas psicológicas: principios, aplicaciones y temas (6 ${ }^{\mathrm{a}}$ ed). México: Thomson.

24. Lane, K., Esser, J., Holte, B., \& McCusker, M. (2010). A study of nurse faculty job satisfaction in community colleges in Florida. Teaching and Learning in Nursing, 5(1), 16-26. doi:10.1016/j.teln.2009.05.001

25. Marqués, L. (2002). La implantación de la gestión de la calidad total en un patronato municipal de deportes. Utilización del modelo EFQM de excelencia empresarial para el sector público. El caso del patronato municipal de deportes del ayuntamiento. (Tesis Doctoral). España. Universidad de Zaragoza.

26. Medina, R., Ceballos, O., Giner, E., \& Marqués, L. (2009). Índice de satisfacción de los trabajadores respecto a su actividad desempeñada en una dirección de deportes municipal. Apunts Educación física y deportes, 96, 96-102.

27. Mendoza, M., Orgambidez, A., \& Carrasco, A. (2010). Orientación a la calidad total, satisfacción laboral, comunicación y compromiso en establecimientos de turismo rural de la Sierra de Huelva. Pasos. Revista de Turismo y Patrimonio Cultural, 8(2), 351-361.

28. Oviedo, H. C., \& Campo-Arias, A. (2005). Aproximación al uso del coeficiente alfa de Cronbach. Revista colombiana de psiquiatría, 34(4), $572-580$.

29. Pelit, E., Öztürk, Y., \& Arslantürk, Y. (2011). The effects of employee empowerment on employee job satisfaction: A study on hotels in Turkey. 
Año 13.

Revista de Investigación

Académica sin Frontera

Núm. 33

ISSN: 2007-8870

https://revistainvestigacionacademicasinfrontera.unison.mx/index.php/RDIASF/issue/view/22

Recibido el 23 de mayo de 2020. Dictaminado mediante arbitraje favorablemente 20 de diciembre de 2020.

International Journal of Contemporary Hospitality Management, 23(6), 784-802.

30. Reyes Robles, M. (2018). La satisfacción de clientes/deportistas y trabajadores en entidades deportivas como determinante de los servicios deportivos (Tesis Doctoral). Universidad Autónoma de Nuevo León. México.

31. Reyes-Robles, M., Medina-Rodríguez, R., Ramírez-Siqueiros, M., LópezWalle, J., \& Ceballos-Gurrola, O. (2018). Satisfaction of an employee in a sports entity: design and validation of a measurement scale. Journal of Physical Education Research, 5(1), 53-63.

32. Rodríguez, J. B. (2016). Condiciones de trabajo, satisfacción laboral y calidad de vida laboral en educación y sanidad (Tesis doctoral). Universidad miguel Hernández de Elche. España.

33. Stringer, C., Didham, J., \& Theivananthampillai, P. (2011). Motivation, pay satisfaction, and job satisfaction of front-line employees. Qualitative Research in Accounting \& Management, 8(2), 161-179. doi 10.1108/11766091111137564 
https://revistainvestigacionacademicasinfrontera.unison.mx/index.php/RDIASF/issue/view/22

Recibido el 23 de mayo de 2020. Dictaminado mediante arbitraje favorablemente 20 de diciembre de 2020.

\section{Directorio Institucional}

Dr. Enrique Fernando Velázquez Contreras

Rector

Dr. Ramón Enrique Robles Zepeda

Secretario General Académico

Dra. Rosa María Montesinos Cisneros

Secretaria General Administrativa

Dr. Rodolfo Basurto Álvarez

Director de Vinculación y Difusión

Dra. Adriana Leticia Navarro Verdugo

Vicerrectora de la Unidad Regional Sur

Dr. Ernesto Clark Valenzuela

Director de la División de Ciencias Económicas y Sociales

Dr. Francisco Espinoza Morales

Secretario de la División de Ciencias Económico y Sociales

Dra. Leticia María González Velásquez

Jefe del Departamento de Ciencias Económico Administrativas

Dra. Lidia Amalia Zallas Esquer

Jefe de Departamento de Ciencias Sociales 
https://revistainvestigacionacademicasinfrontera.unison.mx/index.php/RDIASF/issue/view/22

Recibido el 23 de mayo de 2020. Dictaminado mediante arbitraje favorablemente 20 de diciembre de 2020.

\section{Comité Directivo}

\section{Editor Responsable}

Dr. Francisco Espinoza Morales

Universidad de Sonora

Directora

Dra. Leticia María González Velásquez

Universidad de Sonora

Subdirector

Dr. Javier Carreón Guillen

Universidad Nacional Autónoma de México

Editor Científico

Dr. Cruz García Lirios

Universidad Autónoma del estado de México

\section{Master Gráfico}

M.T.I. Francisco Alan Espinoza Zallas

Universidad Estatal de Sonora

Nos complace anunciar que su diario, "Academic Research Journal Withoutborders" (ISSN/EISSN 2007-8870) fue evaluado positivamente en la indexación Citefactor, ahora la página de la revista está disponible en línea, en caso de cualquier problema.

Journals Master International Innovative Journal Impact Factor (IIJIF)

Red Latinoamericana de revistas Académicas en Ciencias Sociales y Humanidades
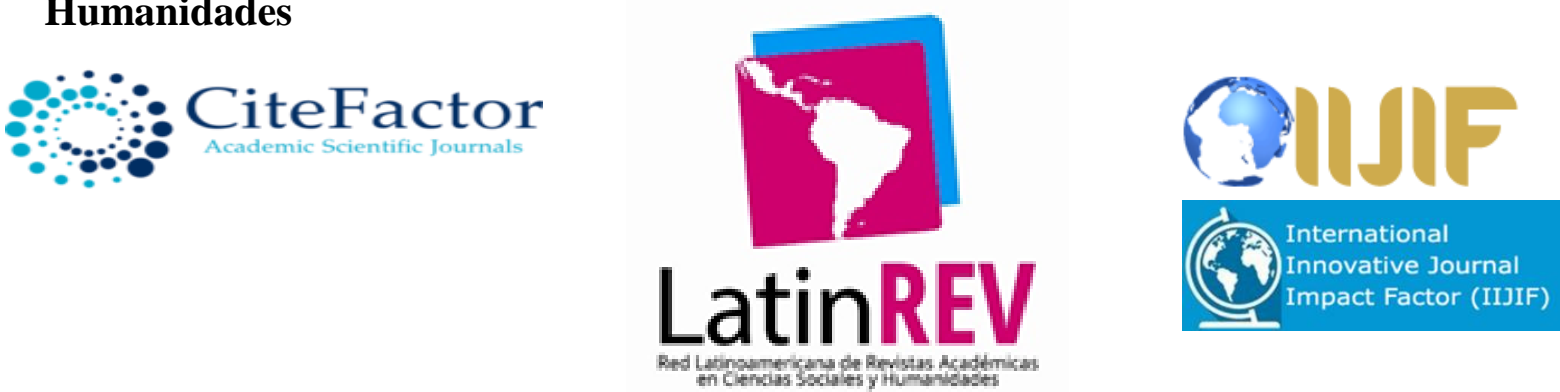
https://revistainvestigacionacademicasinfrontera.unison.mx/index.php/RDIASF/issue/view/22

Recibido el 23 de mayo de 2020. Dictaminado mediante arbitraje favorablemente 20 de diciembre de 2020.

\section{Comité editorial}

Dra. Angélica María Rascón Larios

Universidad de Sonora. México

Dra. María del Rosario Molina González

Universidad de Sonora

Dra. Francisca Elena Rochin Wong

Universidad de Sonora. México

Dra. Lidia Amalia Zallas Esquer

Universidad de Sonora. México

Dra. Beatriz Llamas Arechiga

Universidad de Sonora. México

Dr. Rogelio Barba Álvarez

Universidad de Guadalajara. México

Dra. Rosa María Rincón Ornelas

Universidad de Sonora. México

Dr. Juan Flores Preciado

Universidad de Colima. México

Dr. Amado Olivares Leal. Universidad de Sonora

Universidad de Sonora. México

Dr. Guillermo Velázquez Valadez.

Instituto Politécnico Nacional (IPN) México

Dr. Hugo Nefstalí Padilla Torres.

Universidad Estatal de Sonora. México

Dr. Luis Ramón Moreno Moreno.

Universidad Autónoma de Baja California. México

Dr. Miguel Ángel Vázquez Ruiz.

Universidad de Sonora. México

Dra. Lorena Vélez García.

Universidad Autónoma de Baja California. México 
https://revistainvestigacionacademicasinfrontera.unison.mx/index.php/RDIASF/issue/view/22

Recibido el 23 de mayo de 2020. Dictaminado mediante arbitraje favorablemente 20 de diciembre de 2020.

Dra. Pabla Peralta Miranda.

Universidad Simón Bolívar, Barranquilla, Colombia

\section{Mtro. Roberto Espíritu Olmos}

Universidad de Colima (FCA Tecomán) Colima

\section{Dr. Héctor Priego Huertas.}

Universidad de Colima (FCA Tecomán) Colima

Mtra. María Guadalupe Alvarado Ibarra.

Universidad de Sonora. México.

MSc. Celso Germán Sánchez Zayas

Universidad de Camagüey, Ignacio Agramonte Loynaz, Cuba

Dra. María Luisa Quintero Soto

Universidad Autónoma del Estado de México

Dr. Eyder Bolivar Mojica

Universidad Católica, Luis Amigó, Medellin, Colombia

Revisores de Textos en Inglés

Mtro. Renato Encinas

Mtra. Cecilia Guadalupe Martínez Solano

\section{Comité científico}

Dr. Rosendo Martínez Jiménez. Universidad Autónoma Benito Juárez de Oaxaca.

Dr. Hugo Neftalí Padilla. Universidad Estatal de Sonora

Dra. María Teresa Gaxiola Sánchez. Universidad de Sonora.

Dr. José Cesar Kaplan. Universidad Estatal de Sonora.

Dr. Alfredo Islas Rodríguez. Universidad de Sonora

Frecuencia de publicación: semestral / 2 números por año. 


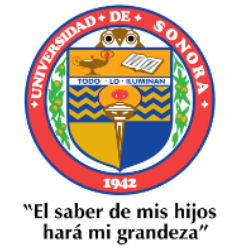

Año 13.

https://revistainvestigacionacademicasinfrontera.unison.mx/index.php/RDIASF/issue/view/22

Recibido el 23 de mayo de 2020. Dictaminado mediante arbitraje favorablemente 20 de diciembre de 2020.

Revista de Investigación Académica sin Frontera (RIASF) con (ISSN: 2007-8870) es un interlocutor internacional de acceso abierto revisado diario en línea en el ámbito del de las Ciencias Económicas Administrativas y Sociales. Su objetivo principal es dar a los trabajos de investigación de calidad. Cubre todas las sub-campos de los campos anteriormente mencionados. Proporciona la plataforma a académicos, estudiantes y profesionales. Sólo pública trabajos de investigación y artículos de revisión inicial. Documento presentado debe cumplir con algunos criterios como, debe ser original, inédita y no estén sometidos a ninguna otra revista.

RIASF es una revista arbitrada / Revisión por pares International. Publicamos documentos sobre una variedad de temas, contextos y estrategias de análisis que examinan la relación entre la rápida evolución para la Sociedad y la tecnología del conocimiento.

REVISTA DE INVESTIGACIÓN ACADÉMICA SIN FRONTERA, Año 13, No. 33, Julio diciembre 2020, es una publicación semestral de investigación científica, editada por la Universidad de Sonora, a través de las División de Ciencias Económicas y Sociales, de la Unidad Regional Sur, Blvd. Lázaro Cárdenas No. 100, Col. Francisco Villa, Navojoa, Sonora, Sonora, México, C.P. 85880. Tel. (642) 425- 99-54.

https://revistainvestigacionacademicasinfrontera.unison.mx/index.php/RDIASF revistaacademicasinfrontera@unison.mx.

Editor responsable: Francisco Espinoza Morales. Reserva de Derechos al Uso Exclusivo: 042013-121811323700-203 e ISSN: 2007-8870, ambos otorgados por el Instituto Nacional de Derecho de Autor. Inscrita en el Directorio de LATINDEX, con Núm. De folio 20014, folio único 14590. Responsable de la última actualización de este Número, Unidad Informática de la Universidad de Sonora, fecha de la última modificación, 30 de diciembre 2020, indexada a Cite Factor Academic Scientific Journal y Journals Master (IIJIF) y Red Latinoamericana de Revistas Académicas en Ciencias Sociales y Humanidades, (Latín Rev). Las opiniones expresadas por los autores no necesariamente reflejan la postura del editor de la publicación. Se autoriza la reproducción total o parcial de los contenidos e imágenes en la presente publicación siempre y cuando se cuente con la autorización del editor y se cite plenamente la fuente. 
Revista de Investigación

Año 13.

Académica sin Frontera

Núm. 33

ISSN: 2007-8870

https://revistainvestigacionacademicasinfrontera.unison.mx/index.php/RDIASF/issue/view/22

Recibido el 23 de mayo de 2020. Dictaminado mediante arbitraje favorablemente 20 de diciembre de 2020.

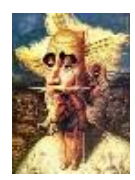

Nos complace anunciar que su diario, "Academic Research Journal Withoutborders"

(ISSN/EISSN 2007-8870) fue evaluado positivamente en la indexación Citefactor, ahora la página de la revista está disponible en línea, en caso de cualquier problema.

Journals Master International Innovative Journal Impact Factor (IIJIF)

Red Latinoamericana de revistas Académicas en Ciencias Sociales y Humanidades

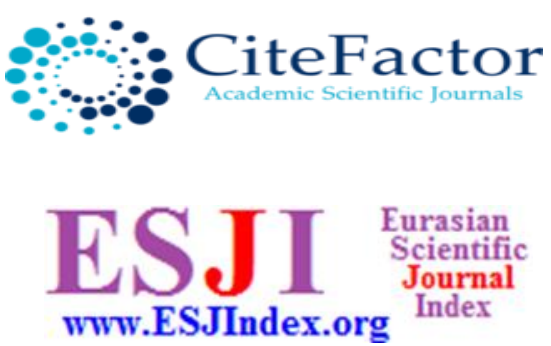

https://www.neliti.com

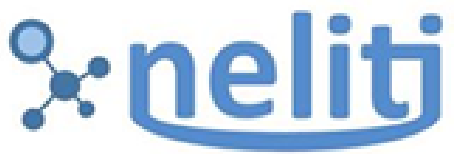

Indonesia's Research Repository

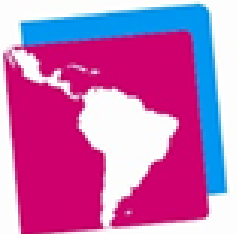

LatinREV

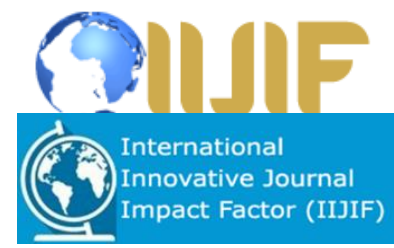

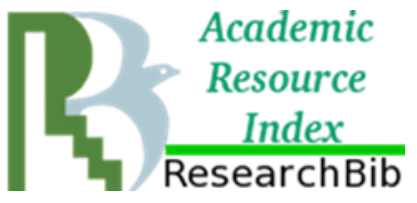

